\title{
Millimetre-sized quasicrystals grown from aluminium alloy
}

\section{Kevin Knowles}

QUASICRYSTALS, materials with 'forbidden' five-fold symmetry, were discovered two years ago, but their investigation has been dogged by the lack of large crystals. The critical X-ray and electron diffraction data which demonstrate five-fold symmetry are from very small quasicrystal particles, complicating interpretation of diffraction intensities which so far do not seem to be consistent with any clear atomic arrangement. But investigations should take a leap forward with the report

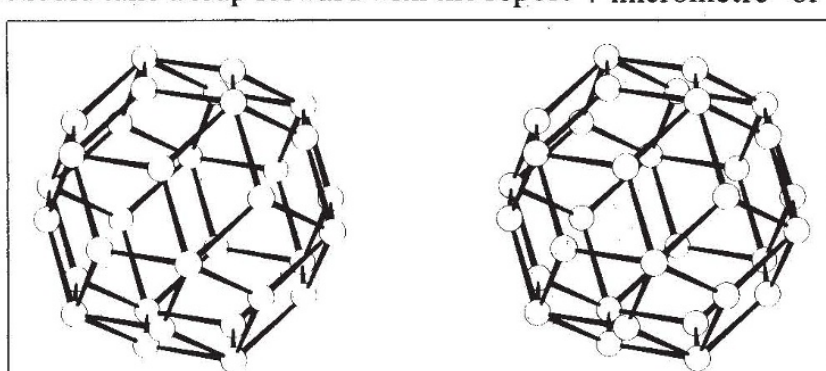

Rhombic triacontahedron, arranged as a stero pair for a threedimensional view (courtesy of A. Mackay, University of London).
The T2 phase is not only of academic interest - it occurs as a stable grain boundary precipitate in commercial $\mathrm{Al}$ $\mathrm{Li}-\mathrm{Cu}-\mathrm{X}$ alloys such as those now being developed by aluminium manufacturers for light aerospace materials with consequent savings in fuel bills. The dimensions of the $\mathrm{Al}-\mathrm{Li}-\mathrm{Cu} \mathrm{T} 2$ grains grown by Dubost et al. under conditions of very slow cooling, allowing only a few nuclei to grow, contrast with the precipitates of micrometre or sub-micrometre dimensions produced from other icosahedral phases, for which the cooling rates needed to produce the phases are typically of the order of $10^{7} \mathrm{~K} \mathrm{~s}^{-1}$, similar to the fastest quench rates reported for obtaining crystalline phases, but far slower than quench rates for metallic glass formation. Indeed, these T2 grains are sufficiently

of Dubost et al. on page 48 of this issue ${ }^{1}$, which describes the growing of quasicrystals approaching a millimetre across, and raises the real prospect that clear $\mathrm{X}$ ray and neutron diffraction pictures of single quasicrystals will now be possible.

One of the basic principles of morphological crystallography is that the external shapes of crystals are determined by the point-group symmetry of the underlying fundamental units of the material, the unit cells, which are typically of nanometre dimensions. Thus most scientists in their schooldays will have grown crystals of alum from solution and will have found that although some will crystallize in regular octahedra characteristic of the underlying cubic symmetry, most look irregular, but nevertheless can be described in terms of simple arrangements of the unit cell.

The quasicrystals of Dubost et al. are grains of an $\mathrm{Al}-\mathrm{Li}-\mathrm{Cu}$ alloy showing triacontahedral morphology. They are 'quasicrystalline' in the sense that their morphology alone would imply an underlying 532 or $m \overline{3} \overline{5}$ point-group symmetry, incompatible with translational symmetry inherent in the perfect crystallline state. The grains grew from the 'T2' phase of $\mathrm{Al}-\mathrm{Li}-\mathrm{Cu}$, one of the family of icosahedral phases whose structure and properties have been of intense experimental and theoretical interest since the initial reports of these phases in alloys of $\mathrm{Al}-\mathrm{Mn}, \mathrm{Al}-\mathrm{Fe}$ and $\mathrm{Al}-\mathrm{Cr}^{2-4}$. large to produce X-ray and neutron diffraction patterns from single crystals. The results from such experiments are keenly awaited, as the size of quasicrystals produced to date has allowed only powder $\mathrm{X}$-ray diffraction data to be obtained.

These results should help to determine the origin of the line broadening in powder $\mathrm{X}$-ray diffraction patterns that can be interpreted as evidence of either frozen-in phason strain or random icosahedral packing ${ }^{5,6}$. (The simplest way to see a phason in quasicrystalline materials is as a local violation in the matching rules in a Penrose tiling ${ }^{7}$, switching, for example, the internal vertices of rhombic triacontahedral units within the tiling.)

The ability to produce large grains of icosahedral phase will also be important for other electron-beam work, which indicates that the chemical composition of icosahedral phases grown under rapid cooling conditions is inhomogeneous on a $5-10-\mathrm{nm}$ scale. Chen et al. ${ }^{8}$ have reported changes in the concentration of $\mathrm{Mn}$ in $\mathrm{Al}-\mathrm{Mn}$ icosahedral phase from place to place, and dark-field transmission electron microscopy reveals cloudy and speckly contrast that can vary as a function of diffraction vector, beam direction and alloy formation $^{9-14}$.

This work implies that structural features analogous to spinodal decomposion or anti-phase domain boundaries in crystalline materials are inherent in rapidly solidified icosahedral phases. Will this also be found to be the case in the large $\mathrm{T} 2 \mathrm{Al}-\mathrm{Li}-\mathrm{Cu}$ grains where presumably equilibrium is more easily reached?

The mechanical properties of this phase are particularly relevant. Theoretical considerations by Lubensky et al. ${ }^{15}$ on the basis of hydrodynamic arguments predict that quasicrystals are inherently extremely brittle as dislocation motion is virtually inhibited by the slowness of phason modes in these materials.

On this basis, Lubensky et al. suggested that it would be virtually impossible to fabricate and manufacture macroscopic quasicrystals (such as the ones grown by Dubost et al.), although they recognized that the dynamics of dislocation motion in quasicrystals would alter radically if the phason modes were rendered non-hydrodynamic. It is therefore of interest to discover whether the T2 phase is indeed extremely brittle or whether it is instead merely similar to other intermetallic phases, which in general are characterized by high hardness and limited ductility. Dislocations, if they exist in icosahedral materials, must, on the basis of current electron microscope evidence, be either extremely rare or otherwise not exhibit the characteristic strainfield contrast seen in crystalline materials.

It seems clear that the report of Dubost et al. will stimulate more work on icosahedral materials, particularly on the thermal stability and energetics of the phase formation in $\mathrm{Al}-\mathrm{Li}-\mathrm{Cu}$. We can only imagine where our understanding of icosahedral materials would be if more than 30 years ago Hardy and Silcock ${ }^{16}$, the original discovers of the $\mathrm{T} 2$ phase, had had an electron microscope and had been able to obtain similar icosahedral electron-diffraction patterns to those found by Shechtman et al. ${ }^{2}$ in $\mathrm{Al}-\mathrm{Mn}$.

1. Dubost, B., Lang, J.-M., Tanaka, M., Sainfort, P. \& Audier, M. Nature 324, 48 (1986).

2. Shechtman, D., Blech, I., Gratias, D. \& Cahn, J.W. Phys. Rev. Lett. 53, 1951 (1984)

3. Mackay, A.L. \& Kramer, P. Nature 316, 17 (1985)

4. Mackay, A.L. Nature 323, 293 (1986).

5. Horn, P.M., Malzfeldt, W., DiVincenzo, D.P., Toner, J. \& Gambino R. Phys. Rev. Lett. 57, 1444 (1986).

6. Stephens, P.W. \& Goldman, A.I. Phys. Rev. Lett. 56, 1168 (1986)

7. Socolar, J.E.S. J. de Physique 47, Coll. C3 p.217 (1986).

8. Chen, C.H., Joy, D.C., Chen, H.S. \& Hauser, J.J. Phys. Rev. Lett. 57, 743 (1986)

9. Shechtman, D. \& Blech, 1.A. Met. Trans. A16, 1005 (1985).

10. Field, R.D. \& Fraser, H.L. Mat. Sci. Engng, 68, L17 (1984-85).

1. Urban, K. et al. J. de Physique 47, Coll C3 P.465 (1986)

12. Knowles, K.M. \& Stobbs, W.M. Nature 323, 313 (1986)

13. Saito, Y., Takemoto, A., Tanaka, T. \& Mihama, K. Proc XIth Int. Cong. Electron Micros. p.523 (1986).

14. Robertson, J.L., Misenheimer, M.E., Moss, S.C. \& Bendersky, L.A. Acta Metallurgica 34, 2177 (1986).

15. Lubensky, T.C., Ramaswamy, S. \& Toner, J. Phys. Rev. B32, 7444; 33, 7715 (1986)

16. Hardy, H.K. \& Silcock, J.M., J. Inst. Metals 84, 423 $(1955-56)$

Kevin Knowles is in the Department of Materials Science and Metallurgy, University of Cambridge, Cambridge CB2 3QZ, UK. 